PROCEEDINGS OF THE

AMERICAN MATHEMATICAL SOCIETY

Volume 116, Number 2, October 1992

\title{
THE FIELD OF DEFINITION OF A REAL REPRESENTATION OF A QUIVER $Q$
}

\author{
AIDAN SCHOFIELD
}

(Communicated by Maurice Auslander)

\begin{abstract}
In answer to a question of Kac, we show that if $\alpha$ is a real root for the quiver $Q$, then there is a unique indecomposable of dimension vector $\alpha$ defined over the rational numbers.
\end{abstract}

Kac [1] showed that over an algebraically closed field the dimension vectors of indecomposable representations form the positive root system of the quadratic form associated to the symmetric generalized Cartan matrix defined by the underlying graph of $Q$. Moreover, there is a unique indecomposable representation whose dimension vector is a real root. He asked whether this remains true over arbitrary fields. His method of proof showed that it is true over fields of characteristic $p$. The purpose of this note is to show that the part for real roots is fairly simple to solve over $\mathbb{Q}$.

We fix the notation for the rest of the paper. $Q$ is a quiver with vertex set $\{v, \ldots\}=Q_{0}$ and arrow set $Q_{1}$. If $R$ is a representation over the field $K, \operatorname{dim}_{K}(R)$ is the dimension vector over $K$. We fix a real root $\alpha$ for the quiver $Q$. The important point that we use is that there is a unique indecomposable representation of $Q$ of dimension vector $\alpha$ over $\overline{\mathbb{Q}}$, the algebraic closure of $\mathbb{Q}$, and no indecomposables of dimension vector $m \alpha, m>1$. Also, h. c. $f_{v}\{\alpha(v)\}=1$.

Lemma 1. Let $R$ be a representation of $Q$ over a field $K$; then $\left.R \otimes_{K} L\right|_{K} \cong R^{n}$ as representations of $Q$ over $K$, where $[L: K]=n$.

Proof. A basis for $L$ over $K$ gives a decomposition of $\left.R \otimes_{K} L\right|_{K}$.

Lemma 2. Let $R$ be an indecomposable representation of $Q$ over a field $K$; then any summand of $R \otimes_{K} L$ as a representation over $L$ has dimension vector some rational multiple of the dimension vector of $R$ over $K$.

Proof. Let $R^{\prime}$ be a summand of $R \otimes_{K} L$; then $\underline{\operatorname{dim}}_{L} R^{\prime}=\frac{1}{n} \underline{\operatorname{dim}}_{K}\left(\left.R^{\prime}\right|_{K}\right)=$ $\frac{1}{n} \underline{\operatorname{dim}}_{K} R^{m}$ by Lemma 1 . Here, $n=[L: K]$.

Lemma 3. Let $R$ and $S$ be indecomposable representations of the quiver $Q$ over a field $K$ of dimension vectors $m \alpha$ and $m^{\prime} \alpha$ respectively where $\alpha$ is a real root. Then $R \cong S$.

Received by the editors March 31, 1989 and, in revised form, September 6, 1990.

1980 Mathematics Subject Classification (1985 Revision). Primary 16A64.

Supported by NSF Grant DMS-880-6371. 
Proof. $\left(R \otimes_{K} \bar{K}\right)^{m^{\prime}} \cong\left(S \otimes_{K} \bar{K}\right)^{m}$ since, by Lemma 2, any summand of $R \otimes_{K} L$ has dimension vector a multiple of $\alpha$, for a finite extension $L$ of $K$ and so by taking limits the same holds for $R \otimes_{k} \bar{K}$, where there is a unique indecomposable of dimension vector a multiple of $\alpha$, the one of dimension vector $\alpha$. This isomorphism must be defined over some finite extension field $L$ of $K$. So,

$$
\left(R \otimes_{K} L\right)^{m^{\prime}} \cong\left(S \otimes_{K} L\right)^{m}
$$

and

$$
\left.\left.\left(R \otimes_{K} L\right)^{m^{\prime}}\right|_{K} \cong\left(S \otimes_{K} L\right)^{m}\right|_{K}:
$$

by Lemma $1, R^{m^{\prime}} \cong S^{m} \Rightarrow m^{\prime}=m$ and $R \cong S$.

Since there is an indecomposable representation of dimension vector $\alpha$ over the algebraic closure of $\mathbb{Q}$, there is some finite dimension extension of $\mathbb{Q}$, $K$, over which this representation is defined. We may take $K$ to be a Galois extension of $\mathbb{Q}$.

Lemma 4. Let $K \supset \mathbb{Q}$ be some finite Galois extension of $\mathbb{Q}$ over which there is an indecomposable representation of dimension vector $\alpha, R$. Then any summand of $\left.R\right|_{\mathbb{Q}}$ has dimension vector over $\mathbb{Q}$ a multiple of $\alpha$.

Proof. Let $T$ be an indecomposable summand of $\left.R\right|_{\mathbb{Q}}$ and $\operatorname{dim}_{\mathbb{Q}} T=\beta$; then $T \otimes_{\mathbb{Q}} K$ is a summand of $\left.R\right|_{\mathbb{Q}} \otimes_{\mathbb{Q}} K$, which is isomorphic to $R \otimes_{K}$ $\left(K \otimes_{\mathbb{Q}} K\right) \cong \bigoplus_{\sigma \in G} R^{\sigma}$, where $G$ is the Galois group of $K$ over $\mathbb{Q}$ and $R^{\sigma}$ is the indecomposable representation over $K$ obtained from $R$ by the automorphism of scalars $\sigma: K \rightarrow K$. Since $\operatorname{dim}_{K} R^{\sigma}=\alpha$, Lemma 2 implies that $\alpha$ is a rational multiple of $\beta$, and since h.c. $f_{v}\{\alpha(v)\}=1$ for a real root $\alpha, \beta=s \alpha$ for some integer $s$.

Lemma 5. Let $R$ be a representation of a quiver $Q$ over a field $K$; then $\operatorname{End}\left(R \otimes_{K} L\right) \cong \operatorname{End}(R) \otimes_{K} L$.

Proof. This is standard. The endomorphism ring over $K$ is the vector subspace of $\operatorname{End}_{K}(R)$, the endomorphism of $R$ as a vector space that commutes with certain operators: the dimension of this is independent of the field we work over, so the result follows.

In Lemma 4, we constructed an indecomposable representation $T$ of dimension vectors $s \alpha$ over $\mathbb{Q}$. Let $\operatorname{End}(T) / \operatorname{rad}(\operatorname{End}(T)) \cong D$ be a division algebra with centre $C \supseteq \mathbb{Q}$.

Lemma 6. In the preceding paragraph, $C=\mathbb{Q}$.

Proof. Let $K \supset C \supset \mathbb{Q}$ be the normal closure of $C$ over $\mathbb{Q}$.

Then $\operatorname{End}\left(T \otimes_{\mathbb{Q}} K\right) \cong \operatorname{End}(T) \otimes_{\mathbb{Q}} K$ by Lemma 5. So

$$
\operatorname{End}\left(T \otimes_{\mathbb{Q}} K\right) / \operatorname{rad} \operatorname{End}\left(T \otimes_{\mathbb{Q}} K\right) \cong \operatorname{End}(T) / \operatorname{rad} \operatorname{End}(T) \otimes_{\mathbb{Q}} K \cong D \otimes_{\mathbb{Q}} K .
$$

But center $\left(D \otimes_{\mathbb{Q}} K\right) \cong C \otimes_{\mathbb{Q}} K$, which is not a field unless $K=C=\mathbb{Q}$.

However, all the summands of $T \otimes_{\mathbb{Q}} K$ are isomorphic by Lemmas 2 and 3, so that $\operatorname{End}\left(D \otimes_{\mathbb{Q}} K\right) / \operatorname{rad} \operatorname{End}\left(D \otimes_{\mathbb{Q}} K\right)$ is a simple algebra.

Hence $K=C=\mathbb{Q}$.

Lemma 7. The notations are as in the preceding lemma. $D=\mathbb{Q}$ and $s=1$.

Proof. Lemma 6 shows $D$ is a central division algebra over $\mathbb{Q}$. Let the index of $D$ be $n$. $D$ embeds in $\operatorname{End}(T)$ since $D$ is a separable algebra over $\mathbb{Q}$, so 
each $R(v)$ is a vector space over $D$ : it follows that $\operatorname{dim}_{\mathbb{Q}} T=s \alpha$ where $n^{2} \mid s$. Let us say, $s=n^{2} m$.

Let $L$ be a maximal subfield of $D$ : then $\operatorname{End}\left(T \otimes_{\mathbb{Q}} K\right) / \operatorname{rad} \operatorname{End}\left(T \otimes_{\mathbb{Q}} K\right)$ $\cong D \otimes_{\mathbb{Q}} K \cong M_{n}(L)$. So $T \otimes_{\mathbb{Q}} L \cong S^{n}$ for some representation $S$ over $L$ : moreover, $\operatorname{End}(S) / \operatorname{rad}$ End $S \cong L$. It follows that $S$ is absolutely indecomposable since $\operatorname{End}\left(S \otimes_{L} \overline{\mathbb{Q}}\right) / \operatorname{rad} \operatorname{End}\left(S \otimes_{\mathbb{Q}} \bar{Q}\right) \cong \overline{\mathbb{Q}} . \operatorname{But} \underline{\operatorname{dim}}_{\overline{\mathbb{Q}}}\left(S \otimes_{L} \overline{\mathbb{Q}}\right)=\underline{\operatorname{dim}}_{L}(S)=$ $n m \alpha$.

However, there is only one indecomposable representation of dimension vector a multiple of $\alpha$, and it has dimension vector $\alpha$, so $n=m=1$, which implies Lemma 7.

Theorem 8. Let $\alpha$ be a real root for the quiver $Q$; then there is a unique indecomposable representation of dimension vector $\alpha$ over the ground field $\mathbb{Q}$. There are no other indecomposable representations of dimension vector a multiple of $\alpha$. Proof. The preceding lemmas show that the representation $T$ constructed just before Lemma 6 is the representation we want. Lemma 3 shows that it is unique.

The same argument works over finite fields to show that there is an indecomposable representation of dimension vector $\alpha$ over $F_{p}$, the field of $p$ elements for $p$ a prime. Lemma 7 is no longer required.

Finally, the following theorem is true with the same proof: we leave it to the reader.

Theorem 9. Let $(Q, R)$ be a quiver with relations over the field $K$. Let $\bar{K} \supset$ $K$ be the separable closure, and let $\alpha$ be a dimension vector for $Q$ such that h. c. $f_{v}\{\alpha(v)\}=1$ and there is a unique indecomposable representation over $\bar{K}$ of dimension vector a multiple of $\alpha$ and this indecomposable representation has dimension vector $\alpha$ over $\bar{K}$.

Then there is a unique indecomposable representation over $K$ of dimension vector a multiple of $\alpha$ and this indecomposable representation has dimension vector $\alpha$ over $K$.

\section{REFERENCES}

1. V. Kac, Infinite root systems, representations of graphs and invariant theory, Invent. Math. 5 (1980), 57-92.

School of Mathematics, UNiversity of BRISTOL, BRISTOL BS8 1TH, UNITED KINGDOM

E-mail address: MA_AS\%CMS.BRISTOL.AC.UK@VTVM2.CC.VT.EDU 